\title{
Levantamento de sintomas depressivos em crianças e adolescentes com hemofilia
}

\author{
A survey of depressive symptoms in children and adolescents with hemophilia
}

\author{
Saint-Clair Bahls, Flávia Rocha Campos Bahls, Juliane Keller, Altamir Sabbag, Ana Elisa \\ Gusso, Débora Carla de Assunção, Maria Fernanda Petry e Roberto Beno Echterhoff
}

\section{Resumo}

Objetivo: Avaliar, através da aplicação do questionário de autoavaliação Children's Depression Inventory (CDI), a presença de sintomas depressivos, ideação suicida e medo da dor em crianças e adolescentes com hemofilia, comparando-os com um grupo-controle sem hemofilia Métodos: Aplicação do CDI em 40 crianças e adolescentes do sexo masculino, com idades entre 7 e 15 anos, sendo 20 com hemofilia e 20 do grupo-controle. Resultados: 0 escore médio do CDI no grupo com hemofilia foi 11,55 $\pm 7,51$ e no grupo-controle, $5,3 \pm 2,7$, diferença estatisticamente significativa $(p=0,0003)$. Ao aplicar o ponto de corte 13 , $35 \%$ do grupo com hemofilia situou-se na faixa indicativa de sintomas depressivos clinicamente relevantes, enquanto no grupo-controle nenhum indivíduo situou-se nessa faixa, diferença que foi estatisticamente significativa $(p=0,008)$. Na análise da presença de ideação suicida, 0 índice do grupo com hemofilia foi $25 \%$ e no grupo-controle $10 \%$, uma diferença estatisticamente considerada não-significativa. $\mathrm{Na}$ análise do medo de sentir dor, o índice no grupo de hemofílicos foi $85 \%$ e no grupo-controle $25 \%$, diferença estatisticamente significativa $(p=0,0002)$. Conclusões: Crianças e adolescentes com hemofilia em nossa amostra clínica apresentam maiores índices de sintomas depressivos e mais medo de sentir dor do que crianças e adolescentes da população geral.

Palavras-chave: crianças e adolescentes, hemofilia, sintomas depressivos, medo de dor.

\begin{abstract}
Objective: To evaluate, using the self-report questionnaire Children's Depression Inventory (CDI), the presence of depressive symptoms, suicidal ideations, and fear of pain in hemophilic children and adolescents, comparing them with a control group without hemophilia. Methods: CDI was applied to 40 male children and adolescents aged 7 to 15,20 patients with hemophilia and 20 of the control group. Results: The total mean score for the hemophilic group in CDI was $11.55 \pm 7.51$, and for the control group, $5.3 \pm 2.7$, a statistically significant difference $(p=0.0003$ ). Using the cut-off score of thirteen, $35 \%$ of the hemophilic group was placed in the range that indicates depressive symptoms, while none of the individuals in the control group was placed in this range, difference statistically significant $(p=0.008)$. In the analysis of the presence of suicidal ideations, the hemophilic group presented $25 \%$ while the control group presented $10 \%$, difference considered non-statistically significant. In the analysis of the fear of pain, the hemophilic group presented $85 \%$ while the control group presented $25 \%$, a statistically significant difference $(p=0.0002)$. Conclusions: Children and adolescents with hemophilia from our sample have a higher rate of depressive symptoms and fear of pain than children and adolescents of the general population.
\end{abstract}

Key words: children and adolescents, hemophilia, depressive symptoms, fear of pain.

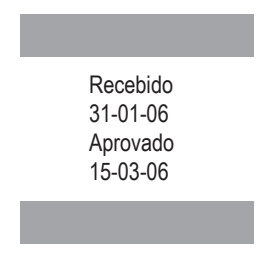

Departamento de Psicologia da Universidade Federal do Paraná (UFPR) (Bahls S-C, Sabbag A,

Gusso AL, Assunção DC, Petry MF, Echterhoff RB)

Universidade Tuiuti do Paraná (UTP) (Bahls FRC, Keller J)

Correspondência para: Saint-Clair Bahls

Rua Carneiro Lobo, 570/1403 - 80240-240 - Curitiba-PR - Telefax: (41) 3242-6132

-e-mail: scbahls@superig.com.br 


\section{Introdução}

A presença de sintomas depressivos é uma complicação comum em pacientes com doenças crônicas e costuma provocar um impacto negativo no curso e no tratamento da doença primária, muitas vezes aumentando os índices de mortalidade. Os sintomas depressivos provocam uma percepção pessimista dos acontecimentos, favorecem uma visão distorcida em relação à doença e influenciam negativamente o cuidado com o tratamento. $\mathrm{Na}$ presença de sintomas depressivos a resposta imunológica diminui, favorecendo infecções e complicando a evolução da patologia clínica de base (Almeida e Meleiro, 2000).

Pacientes com doenças crônicas são considerados grupo de risco para apresentar sintomas depressivos e sua presença costuma interferir desfavoravelmente na evolução clínica (Bahls, 2002a; 2004).

Segundo Van Houdenhove e Onghena (1997) a dor e a depressão podem ser consideradas condições graves de sofrimento humano, sendo que pacientes com dores persistentes têm um risco maior para apresentar sintomas depressivos.

Na Noruega, uma pesquisa (Aass et al., 1997) investigou a presença de sintomas depressivos em pacientes portadores de câncer, com idades entre 10 e 91 anos, e identificou que a presença da dor correlacionou-se positivamente com a incidência de sintomas depressivos. Crianças com doenças físicas crônicas são duas vezes mais suscetíveis de desenvolver um transtorno psiquiátrico quando comparadas às crianças saudáveis (York e Hill, 1997), porém, pouco se conhece sobre a relação entre sintomas depressivos e doenças crônicas na população pediátrica.

Especificamente a respeito da investigação de sintomas depressivos em crianças e adolescentes com hemofilia, em revisão bibliográfica na literatura nacional nada foi obtido e na literatura internacional somente um artigo foi encontrado.

Celiker et al. (2000) pesquisaram na Turquia 47 crianças portadoras de doença clínica crônica (12 com hemofilia e 35 com deficiência física causada pela poliomielite), sendo também empregado um grupo-controle de 36 crianças estudantes saudáveis. A pesquisa utilizou o Children's Depression Inventory (CDI) como instrumento de avaliação. Os escores médios obtidos em cada grupo foram, respectivamente, com poliomielite $10,57 \pm 5,87$, com hemofilia $11 \pm 5,64$ e controle $8,39 \pm 3,78$, não havendo diferença estatisticamente significativa entre os três grupos. Ao empregar o ponto de corte 19, que identifica a presença de sintomas depressivos, o resultado foi $11,4 \%$ para o grupo com poliomielite, $16 \%$ para o com hemofilia e $0 \%$ para o grupo-controle.

Os objetivos da atual pesquisa foram determinar a presença de sintomas depressivos, ideação suicida e preocupação com a dor em crianças e adolescentes hemofílicos, comparando-os com um grupo-controle pareado da população geral. Temos como hipóteses de pesquisa que o grupo de pacientes com hemofilia apresentará significativamente mais sintomas depressivos, ideação suicida e preocupação com a dor do que o grupo-controle. A relevância desse estudo reside no fato de que praticamente não há investigações sobre sintomas depressivos em crianças e adolescentes com doenças físicas crônicas e limitantes.

\section{Métodos}

Foram avaliada 20 crianças e adolescentes, com idades variando entre 7 e 15 anos, do sexo masculino, em tratamento na Associação Paranaense dos Hemofílicos (Centro de Hematologia e Hemoterapia do Paraná [HEMEPAR]), na cidade de Curitiba. A maioria das crianças e adolescentes que lá se encontram vem de outras cidades do Paraná e de outros estados do Brasil. Elas recebem alimentação e transporte até o HEMEPAR para que possam receber tratamento (fator de coagulação), atendimento psicológico, aulas de musicoterapia e atendimento fisioterápico. Todas estão em fase de manutenção da doença, ou seja, fazem fisioterapia diariamente e tomam 0 fator de coagulação quando necessário.

O protocolo foi aprovado pelo comitê de ética em pesquisa da instituição onde foi realizada a pesquisa. Visto que todos os participantes da pesquisa são menores de idade, foi solicitado aos pais e/ou seus responsáveis que assinassem o termo de consentimento da pesquisa. Assim sendo, somente após o consentimento formal deu-se início à aplicação do questionário.

O grupo-controle foi composto por 20 crianças e adolescentes do sexo masculino, entre 7 e 15 anos de idade, provenientes de escolas da rede pública de Curitiba, selecionados através de sorteio. A parte da amostra referente a crianças de 7 a 9 anos foi retirada da amostra não-probabilística coletada por Menegatti (2002) e a referente a crianças e adolescentes de 10 a 15 anos foi retirada da amostra não-probabilística coletada por Bahls (2000). O pareamento se deu em função do sexo (todos eram do masculino) e da idade. A idade média no grupo de pacientes com hemofilia foi $11,4 \pm 4,37$ e no controle, de $11,9 \pm 2,34$, sem diferença estatística $(p=0,5352)$.

0 instrumento utilizado foi o questionário de auto-avaliação CDI, validado e modificado para estudantes brasileiros (Bahls, 2000; Gouveia et al., 1995). O CDI surgiu nos EUA em 1977 e sua última edição é de 1992. Ele mede sintomas depressivos em crianças e adolescentes escolares de 7 a 17 anos e também diferencia crianças e adolescentes com o diagnóstico de depressão maior ou distimia daqueles portadores de outros transtornos psiquiátricos ou normais (Kovacs, 1992).

Vários estudos têm avaliado sua consistência interna, fatores estruturais, confiabilidade, teste e reteste (estabilidade), métodos de administração e escores de ponto de corte para delinear casos de depressão, sendo o coeficiente alfa de Cronbach 0,86 para os 27 itens em amostras normativas. É importante destacar que o CDI não é um instrumento diagnóstico, pois simplesmente mede sintomas depressivos.

Já foram encontradas correlações positivas e significativas entre o diagnóstico clínico e os escores do CDI, tendo sido este validado em amostras comunitárias e clínicas por vários autores (Chartier, Lassen, 1994; Goveia et al., 1995; Kovacs, 1985; 1992; Reynolds et al., 1985; Smucker et al., 86). Sua autora recomenda dois diferentes pontos de corte para avaliar a presença de sintomas depressivos (Kovacs, 1985; 1992). O ponto de corte de 13 deve ser aplicado em amostras clínicas e o 19, em amostras comunitárias. No seu item 9, avalia a presença de ideação suicida, apresentando três pontuações de resposta, da seguinte maneira: (0). "eu não penso em me matar"; (1). "eu penso em me matar, mas 
não o faria"; e (2). "eu quero me matar". E no item 19, avalia a presença do medo de sentir dor, apresentando igualmente três pontuações de resposta: (0). "eu não tenho medo de sentir dor"; (1). "eu quase sempre tenho medo de sentir dor"; e (2). "eu sempre tenho medo de sentir dor".

A aplicação foi realizada de forma individual em uma sala onde 0 aplicador garantiu o tratamento sigiloso e 0 anonimato dos dados fornecidos, esclarecendo também as dúvidas que surgiram em relação ao preenchimento dos dados de identificação. Em seguida, o questionário era lido em voz alta e cada item era consecutivamente respondido pelos participantes. 0 tempo médio para responder 0 instrumento foi de 15 a 20 minutos.

\section{Análise estatística}

Para comparação dos grupos em relação aos escores, considerou-se o teste não-paramétrico de Mann-Whitney; para comparação dos grupos em relação aos pontos de corte 13 e 19 e em relação às questões dos itens 9 e 19, considerouse o teste exato de Fisher; para comparação dos grupos em relação às idades foi considerado o teste $t$ de Student para amostras independentes. Valores de $p<0,05$ indicaram significância estatística.

\section{Resultados}

\section{Presença de sintomas depressivos \\ - Escores totais médios}

Crianças e adolescentes com hemofilia apresentaram escore médio de 11,55 $\pm 7,51$ e as crianças do grupocontrole, $5,3 \pm 2,7$, diferença estatisticamente significativa $(p=0,0003)$.

\section{corte 13 e 19}

- Índice de sintomas depressivos nos pontos de

Ao aplicar o ponto de corte 13 a taxa de sintomas depressivos no grupo com hemofilia foi $35 \%$ e no controle $0 \%$, diferença estatisticamente significativa $(p=0,008)$. Por outro lado, empregando o ponto de corte 19 , a presença de sintomas depressivos no grupo com hemofilia foi $10 \%$ e no controle, $0 \%$, sem diferença estatisticamente significativa $(p=0,2436)$. Esses resultados são apresentados na Tabela 1.

Tabela 1. Freqüência de indivíduos com sintomas depressivos nos pontos de corte 13 e 19 do CDI

\begin{tabular}{|c|c|c|c|c|c|c|c|c|}
\hline & \multicolumn{4}{|c|}{ Ponto de corte 13} & \multicolumn{4}{|c|}{ Ponto de corte 19} \\
\hline & \multicolumn{2}{|c|}{ Hemofílicos } & \multicolumn{2}{|c|}{ Controle } & \multicolumn{2}{|c|}{ Hemofílicos } & \multicolumn{2}{|c|}{ Controle } \\
\hline & Total & $\%$ & Total & $\%$ & Total & $\%$ & Total & $\%$ \\
\hline$(<)$ & 13 & 65 & 20 & 100 & 18 & 90 & 20 & 100 \\
\hline$(\geq)$ & 7 & 35 & 0 & 0 & 2 & 10 & 0 & 0 \\
\hline Total & 20 & 100 & 20 & 100 & 20 & 0 & 20 & 100 \\
\hline$p$ & \multicolumn{4}{|c|}{0,0083} & \multicolumn{4}{|c|}{0,2436} \\
\hline
\end{tabular}

\section{Presença de ideação suicida}

$\mathrm{Na}$ análise do item 9 do $\mathrm{CDI}$, que verifica a presença de ideação suicida, o índice no grupo com hemofilia foi 25\% e no controle, $10 \%$, sem diferença estatisticamente significativa $(p=0,2037)$. Esses resultados encontram-se na Tabela 2.

\section{Presença de medo de sentir dor}

$\mathrm{Na}$ análise do item 19 do CDI, relativo ao medo de sentir dor, o índice no grupo com hemofilia foi $85 \%$ e no controle, $25 \%$, diferença estatisticamente significativa $(p=0,0002)$. Esses resultados encontram-se na Tabela 2.

Tabela 2. Resposta dos indivíduos nos itens 9 e 19 do CDI

\begin{tabular}{|c|c|c|c|c|c|c|c|c|}
\hline \multirow[b]{3}{*}{ Respostas } & \multicolumn{4}{|c|}{$\begin{array}{c}\text { Item } 9 \\
\text { Ideação suicida }\end{array}$} & \multicolumn{4}{|c|}{$\begin{array}{c}\text { Item } 19 \\
\text { Medo de sentir dor }\end{array}$} \\
\hline & \multicolumn{2}{|c|}{ Hemofilicos } & \multicolumn{2}{|c|}{ Controle } & \multicolumn{2}{|c|}{ Hemofilicos } & \multicolumn{2}{|c|}{ Controle } \\
\hline & Total & $\%$ & Total & $\%$ & Total & $\%$ & Total & $\%$ \\
\hline 0 & 15 & 75 & 18 & 90 & 3 & 15 & 15 & 75 \\
\hline 1 ou 2 & 5 & 25 & 2 & 10 & 17 & 85 & 5 & 25 \\
\hline Total & 20 & 100 & 20 & 100 & 20 & 0 & 20 & 100 \\
\hline$p$ & \multicolumn{4}{|c|}{0,2037} & \multicolumn{4}{|c|}{0,0002} \\
\hline
\end{tabular}

\section{Discussão}

O escore médio de sintomas depressivos encontrados em nosso estudo em crianças e adolescentes portadores de hemofilia foi significativamente maior do que em crianças e adolescentes do grupo-controle, indicando presença importante de características depressivas nesses pacientes pediátricos.

$O$ único artigo (Celiker et al., 2000) encontrado na literatura que também investigou sintomas depressivos em crianças e adolescentes com hemofilia empregou o mesmo instrumento de avaliação e obteve escore médio $(11 \pm 5,64)$ semelhante ao deste estudo (11,55 $\pm 7,51)$. Todavia, diferentemente de nossos resultados, não houve diferença estatística em relação ao grupo-controle.

Na pesquisa de Celiker et al. (2000) o escore médio do grupo-controle foi $8,39 \pm 3,78$, enquanto em nosso estudo foi $5,3 \pm 2,7$. Uma provável explicação para essa diferença pode ser o fato de que na pesquisa de Celiker et al. (2000) o grupo-controle não foi exatamente pareado com o grupo hemofílico como em nosso estudo. Havia 12 crianças e adolescentes no grupo hemofílico e 36 no grupo-controle e, como o número de participantes no estudo foi pequeno, a diferença em relação ao grupo-controle pode ter sido prejudicada na análise estatística.

Duas outras pesquisas avaliaram, em crianças e adolescentes portadores de câncer, a presença de sintomas depressivos aplicando o mesmo instrumento (CDI). Noll et al. (1999), nos EUA, investigaram um grupo de 76 jovens com idades entre 8 e 15 anos em quimioterapia e obtiveram o escore médio de 7,42 $\pm 6,61$. Von Essen et al. (2000), na Suécia, em 51 crianças e adolescentes, 
com idades entre 8 e 18 anos, sendo 16 em tratamento e 35 já fora do esquema de tratamento (considerados sobreviventes da doença), encontraram escore médio de $7 \pm 5,4$ no grupo em tratamento e de 8,6 \pm 6,5 no grupo sobrevivente.

Os escores médios de sintomas depressivos em crianças e adolescentes com câncer são semelhantes nas duas pesquisas, porém menores do que os encontrados por nós em crianças e adolescentes com hemofilia. Esses resultados sugerem diferentes reações quanto à presença de sintomas depressivos nessas duas populações pediátricas. É possível que crianças e adolescentes com hemofilia apresentem menos resiliência no enfrentamento da evolução da patologia clínica, o que favorecia o aparecimento de sintomas depressivos.

A maneira como crianças e adolescentes reagem emocionalmente aos desdobramentos de suas doenças clínicas crônicas tem sido objeto de estudo nos últimos anos, parecendo que algumas populações têm mais capacidade do que outras (Arrais e Araújo, 1999; Noll et al., 1999; York, Hill, 1997). Entretanto, mais pesquisas são necessárias para um melhor esclarecimento sobre esse importante aspecto.

Em nosso estudo com o ponto de corte 13, os sintomas depressivos foram identificados em $35 \%$ dos participantes do grupo com hemofilia versus $0 \%$ no grupo-controle, diferença estatisticamente significativa; no ponto de corte 19, no grupo com hemofilia $10 \%$ apresentaram sintomas depressivos contra $0 \%$ no controle, sem diferença estatisticamente significativa.

Resultados semelhantes foram obtidos no estudo turco de Celiker et al. (2000), que aplicou o ponto de corte 19 e identificou a presença de $16 \%$ de sintomas depressivos no grupo com hemofilia contra $0 \%$ no controle.

Outras pesquisas que utilizaram o CDI para avaliar sintomas depressivos, em crianças e adolescentes com doenças clínicas, obtiveram os seguintes resultados: o estudo sueco de Von Essen et al. (2000), aplicando o ponto de corte 13 em população pediátrica (8 a 18 anos de idade) com câncer, encontrou $14 \%$ da amostra com sintomas depressivos, sendo a maioria representada pelos sobreviventes ao tratamento. A pesquisa turca de Çavusoglu (2001) aplicou o ponto de corte 19 , também em população pediátrica ( 9 a 13 anos de idade) com câncer, e encontrou $22 \%$ da amostra com sintomas depressivos.

Em crianças e adolescentes brasileiros hospitalizados devido a doenças agudas e cônicas (6 a 15 anos de idade), Bandim et al. (1998), no ponto de corte 18, identificaram $40 \%$ com sintomas depressivos. É importante considerar que, mesmo empregando um único instrumento de avaliação, as pesquisas apresentam diferenças metodológicas que recomendam cautela na análise comparativa de seus resultados. Pode-se, todavia, destacar que crianças e adolescentes com doenças clínicas crônicas representam uma população de risco para a presença de sintomas depressivos, com índices, medidos pelo CDI, variando entre $10 \%$ e $40 \%$.

Ainda sobre os resultados encontrados em nosso estudo, observamos que aplicando um ponto de corte mais baixo (13) a presença de sintomas depressivos foi alta (35\%) e significativamente diferente do grupo-controle, mas, no ponto de corte maior (19), não ocorreu diferença em relação ao grupo-controle. Isso nos permite supor que a população pediátrica portadora de hemofilia não tende a apresentar sintomas depressivos de intensidade grave, porém a presença de sintomas depressivos de intensidade moderada parece ser comum, indicando maior necessidade de atenção na identificação do quadro por parte dos cuidadores dessas crianças e adolescentes. Se a sintomatologia é menos grave, tem maior possibilidade de passar despercebida, ocasionando dificuldades que poderiam ser evitadas no tratamento e na evolução da patologia de base.

Quanto à avaliação de ideação suicida, nosso estudo não se encontrou diferença significativa entre o grupo com hemofilia $(25 \%)$ e o controle (10\%), indicando que a presença de doença hemofílica não é fator de risco para esse tipo de sintoma. Apesar de um quarto dos participantes do grupo com hemofilia pontuar a presença de pensamentos suicidas, esse índice não foi superior ao grupo-controle pareado, o que pode ser explicado pelo fato de que pensamentos suicidas são, infelizmente, presença comum em crianças e principalmente em adolescentes da população geral, como já está bem descrito na literatura nacional (Bahls et al., 2003) e internacional (Chartier e Lassen, 1994; Gunnel, 2000; Kessler et al., 1999).

Também procuramos avaliar em nosso estudo a questão relativa à dor nessa população pediátrica e identificamos uma alta relação entre a presença da doença hemofílica e 0 medo de sentir dor, que ocorreu em $85 \%$ dos participantes do grupo com hemofilia contra $25 \%$ no controle, diferença estatisticamente significativa. Não encontramos na literatura nenhum estudo semelhante em população pediátrica, apesar da compreensão da existência de uma íntima relação entre doenças crônicas, sintomas depressivos e dor (Aass et al., 1997; Silva, 2002; Van Houdenhove e Onghena, 1997).

A hipótese levantada para justificar esses valores obtidos relaciona-se com o fato de que crianças e adolescentes hemofilicos passam por tratamento durante toda a sua vida, visto que a hemofilia é uma doença que não tem cura, sendo passível apenas de controle. Esses jovens podem ter conseqüências sérias decorrentes de leves e pequenos traumas que venham a sofrer: edemas, hemorragias e dificuldade em mobilizar alguns membros. Portanto, a criança hemofílica tem mais dor e sofre mais com as conseqüências de traumas do que uma criança saudável. Essa condição desfavorável parece propiciar uma experiência de maior expectativa negativa e de medo em relação a possíveis reações, tanto aos traumas tão comuns da infância como às intervenções terapêuticas necessárias ao acompanhamento da patologia e seus desdobramentos clínicos.

Apontamos, a seguir, algumas limitações dessa pesquisa. 0 número pequeno de nossa amostra reduz o poder da análise estatística. A presença de participantes somente do sexo masculino traz dificuldade no sentido das comparações com pesquisas que investigaram ambos os sexos como, por exemplo, jovens com câncer, pois sabemos que os meninos costumam pontuar menos sintomas depressivos que as meninas (Bahls, 2002b). Devido ao pequeno número de participantes também não foi possível avaliar a existência ou não de diferenças entre os diversos graus da doença hemofílica, assim como investigar separadamente crianças e adolescentes. 
O emprego de somente um instrumento de avaliação que não foi originalmente desenvolvido para a avaliação desse tipo de população (crianças e adolescentes com doenças clínicas crônicas) pode trazer questionamentos quanto à consistência dos achados. No levantamento sobre ideação suicida e medo de sentir dor, a investigação baseou-se em somente um item do questionário para cada um desses aspectos clínicos, o que pode indicar um pequeno valor em sua capacidade de precisão. Porém, já foi destacado (Bahls et al., 2003; Brown et al., 2003) que, em relação à ideação suicida, estudos têm encontrado, mesmo em escalas de avaliação com um só item, que sua indicação representa um preditor bastante confiável. Recomendamos, em função dessas limitações, cautela na interpretação e na generalização dos achados dessa pesquisa.

\section{Conclusões}

Foram confirmadas as hipóteses de pesquisa de que o grupo com crianças e adolescentes hemofílicos apresenta significativamente mais sintomas depressivos e preocupação com a dor do que o grupo-controle. Os sintomas depressivos identificados situam-se na faixa de intensidade sintomatológica moderada, não sendo confirmada a hipótese de pesquisa de que o grupo com crianças e adolescentes hemofílicos apresenta significativamente mais ideação suicida do que 0 grupo-controle.

A investigação de características depressivas em populações pediátricas portadoras de doenças clínicas crônicas apresenta relevância clínica, uma vez que a presença dessas características tem a possibilidade de complicar de forma importante a evolução e o desfecho do tratamento. Apesar do entendimento de que a presença de sintomas depressivos é esperada nessa população clínica, observamos que a literatura específica sobre esse assunto ainda se baseia em poucos estudos, não devendo ser vista como conclusiva.

Pela sua importância clínica, recomendamos o cuidado na constante avaliação de sintomas depressivos em crianças e adolescentes com patologias crônicas, e, como levantamos a hipótese de que a presença de sintomas depressivos pode variar de acordo com diferentes categorias de doenças crônicas em crianças e adolescentes, sugerimos a realização de pesquisas que avaliem essa relação.

\section{Referências}

Aass N, Fossa SD, Dahl A, Moe TJ. Prevalence of anxiety and depression in cancer patients seen at the Norwegian Radium Hospital. Eur Cancer, 33(10): 1597-604, 1997.

Almeida MA, Meleiro AMAS. Depressão e insuficiência renal crônica: uma revisão. J Bras Nefrol, 22(1): 21-9, 2000.

Arrais AR, Araújo TCCF. Recidiva versus cura: a vivência paradoxal da sobrevivência ao câncer na infância. Rev Bras Cancerol, 45(3): 15-22, 1999

Bahls SC. Sintomas depressivos em estudantes de 10 a 17 anos: um levantamento epidemiológico. Dissertação. Curitiba: UFPR, 2000

Bahls SC. Aspectos clínicos da depressão na infância e adolescência. J Pediatr, 78(5): 359-66, 2002a.

Bahls SC. Epidemiology of depressive symptoms in adolescents from a public school in Curitiba, PR. Rev Bras Psiquiatr, 24(2): 63-7, 2002b.

Bahls SC. A depressão em crianças e adolescentes e o seu tratamento. São Paulo: Lemos Editorial; 2004.

Bahls SC, Keller APR, Moura CR, Zanona M, Sakiyama RR. Ideação suicida em adolescentes de uma escola pública de Curitiba-PR. Psiq Biológica, 11(3): 85-90, 2003.

Bandim JM, Sougey EB, Fonseca L, Tomita TK, Adrião CG. Cuestionario de depresión infantil - CDI: una investigación preliminar con niños hospitalizados. Neurobiol, 61(1): 1-8, 1998.

Brown GS, Jones ER, Betts E, Wu J. Improving suicide risk assessment in a managed-care environment: crisis. J Crisis Interv Suicide Prevention, 24(2): 49-55, 2003.

Celiker R, Kutsal YG, Oy B, Onur O, Gurgey A. Depression in children with hemophilic arthropathy and poliomyelitis: a preliminary report. Turk J Pediatr, 42(1): 27-30, 2000.
Chartier GM, Lassen MK. Adolescent depression: children's depression inventory norms, suicidal ideation, and (weak) gender effects. Adolescence, 29(116): 857-64, 1994

Çavusoglu H. Depression in children with cancer. J Pediatr Nurs, 16 (5): 380-5, 2001.

Gouveia VV, Barbosa GA, Almeida HJF, Gaião AA. Inventário de depressão infantil - CDI: estudo de adaptação com escolares de João Pessoa. J Bras Psiquiatr, 44: 345-9, 1995.

Gunnel DJ. The epidemiology of suicide. Int Rev Psychiatry, 12 : 21-6, 2000.

Kessler RC, Borges G, Walters EE. Prevalence and risk factors for lifetime suicide attempts in the National Comorbidity Survey. Arch Gen Psychiatry, 55: 617-26, 1999.

Kovacs M. The Children's Depression Inventory (CDI). Psychopharmacol Bull, 21(4): 995-8, 1985.

Kovacs M. The Children's Depression Inventory, CDI. Manual MultiHealth Systems, Inc. Toronto, 1992.

Menegatti CL. Interações pais-filhos e depressão infantil: uma abordagem comportamental. Dissertação. Curitiba: UFPR, 2002.

Noll RB, Gartstein MA, Vannata K, Correl J, Bukowski WM, Davies WH. Social, emotion, and behavioral functioning of children with cancer. Pediatrics, 103(1): 71-7, 1999.

Reynolds WM, Anderson G, Bartell N. Measuring depression in children: a multimethod assesment investigation. J Abnorm Psychol, 13: 513-26, 1985.

Silva RMG. Dor na infância. Ars Curandi, 35(8): 56-61, 2002.

Smucker MR, Craighead WE, Craighead LW, Green BJ. Normative and reliability data for the Children's Depression Inventory. J Abnorm Child Psychol, 14: 25-39, 1986. 
Van Houdenhove B, Onghena P. Pain and depression. In: Robertson MM, Katona CLE, editores. Depression and physical illness. West Sussex: Wiley, 1997, p. 465-97.

Von Essen L, Enskär K, Kreuger A, Larsson B, Sjöden PO. Selfesteem depression and anxiety among Swedish children and adolescents on and off cancer treatment. Acta Paediatr, 89: 229-36, 2000.

York A, Hill P. Depression and physical illness in children and adolescence. In: Robertson MM, Katona CLE, editores. Depression and physical illness. West Sussex: Wiley, 1997, p. 145-68. 